\title{
Evaluation of the Performance of an Automated Chemiluminescent Method (Access Beckman Coulter) for Determination of Salivary Cortisol and Clinical Utility
}

\author{
Maria Alejandra Kloberdanz ${ }^{1}$, , Lucia Fernandez ${ }^{1}$, Agustina Peverini ${ }^{1}$, Fernando Smithuis ${ }^{1}$, \\ Adrian Aymard ${ }^{1}$, Claudio Aranda ${ }^{1}$, Bibiana Fabre ${ }^{2}$, Martin Repetto ${ }^{1,2}$, Adriana Oneto, ${ }^{1,2}$ \\ ${ }^{1}$ Laboratory of Clinical Analysis of Buenos Aires (TCba-LACba), CABA, Argentina \\ ${ }^{2}$ Clinical Biochemistry Department, Faculty of Pharmacy and Biochemistry, INFIBIOC, University of Buenos Aires, Buenos Aires, CABA, \\ Argentina
}

Email address:

maraleklo@hotmail.com (M. A. Kloberdanz)

${ }^{*}$ Corresponding author

To cite this article:

María Alejandra Kloberdanz, Lucía Fernández, Agustina Peverini, Fernando Smithuis, Adrián Aymard, Claudio Aranda, Bibiana Fabre, Martín Repetto, Adriana Oneto. Evaluation of the Performance of an Automated Chemiluminescent Method (Access Beckman Coulter) for Determination of Salivary Cortisol and Clinical Utility. American Journal of Clinical and Experimental Medicine.

Vol. 7, No. 6, 2019, pp. 130-134. doi: 10.11648/j.ajcem.20190706.12

Received: October 29, 2019; Accepted: November 20, 2019; Published: December 6, 2019

\begin{abstract}
The diagnostic procedure for the evaluation of Cushing's syndrome is performed by any of the following biochemical tests: urine free cortisol, salivary cortisol at $11 \mathrm{pm}$ and serum cortisol post $1 \mathrm{mg}$ of dexamethasone. Collection of saliva samples is simple and noninvasive, thus being a method of choice for the evaluation of risk populations. The aim of this work is to analyze the performance of an automated chemiluminescent method for measurement of salivary cortisol at $11 \mathrm{pm}$ according to the new quality guidelines and assess its clinical utility. Cortisol levels were measured in samples obtained by passive drooling from 32 healthy subjects and 9 patients with Cushing's syndrome. Matrix effect, linearity, limit of blank, limit of quantitation, recovery and diagnostic performance were assessed. The Unicel 600 DXI Access Beckman Coulter chemiluminescent automated analyzer was used. The standard curve provided by the manufacturer was adapted to measure cortisol concentrations in saliva. Matrix effect: equation of the curve using salivary matrix: $y=-1.824 x+3.491(95 \% \mathrm{CI}=-2.068$ to -1.582$)$ vs. Equation of the curve using diluent matrix: $y=-1.833 \mathrm{x}+3.394$ (95\% CI=-1.961 to -1.704$)$. There is overlapping of both curves. Linearity: linear assay between $1.8 \mathrm{nmol} / \mathrm{L}$ and $108.0 \mathrm{nmol} / \mathrm{L}$. Limit of blank: $0.1 \mathrm{nmol} / \mathrm{L}$. Limit of quantitation: $1.8 \mathrm{nmol} / \mathrm{L}$ (TAE of $25 \%$ ). Recovery: standard cortisol solution concentration $5 \mathrm{nmol} / \mathrm{L}: 102 \%$; $10 \mathrm{nmol} / \mathrm{L}: 107 \%$; $40 \mathrm{nmol} / \mathrm{L}$ : $115 \%$. Diagnostic performance: median and ranges in healthy subjects: $2.0 \mathrm{nmol} / \mathrm{L}(<2.0-9.0 \mathrm{nmol} / \mathrm{L})$; Cushing's syndrome: $30.3 \mathrm{nmol} / \mathrm{L}$ (15.4-61.0 nmol/L). ROC curve cutoff value: $9.0 \mathrm{nmol} / \mathrm{L}$ (100\% Specificity; $100 \%$ Sensitivity; AUC=1.00). The method used provides excellent analytical performance for cortisol measurement in saliva at $11 \mathrm{pm}$, which makes it a valuable biochemical tool both for screening populations at risk for Cushing's syndrome and for the follow-up and diagnosis of this condition.
\end{abstract}

Keywords: Salivary Cortisol, Cushing's Syndrome, Analytical Performance

\section{Introduction}

Diagnostic procedures for the evaluation of Cushing's syndrome (CS) include the following biochemical tests: 24hour urinary free cortisol (UFC) in two samples, serum cortisol measurement after oral administration of $1 \mathrm{mg}$ of dexamethasone and salivary cortisol (Csal) determination in two samples collected at $11 \mathrm{pm}$ based on the Endocrine Society Guidelines [1]. For 24-hour urinary free cortisol measurement, we found some limitations such as the quality of the sample associated with sampling difficulties and inconvenience for the patient. With the dexamethasone suppression test, individual variations or pharmacological 
interferences may occur, which may lead to inconsistent results, especially in patients with obesity, chronic conditions or psychiatric disorders [2-3]. Saliva is produced by the salivary glands, contains basically water, various proteins, enzymes, glycoproteins, carbohydrates and electrolytes. In recent years, a large number of studies have supported the use of saliva for the evaluation of free steroid hormones. Saliva is a natural ultrafiltrate of blood where non-proteinbound steroids diffuse freely [4]. The biologically active free fraction of cortisol in serum is in equilibrium with cortisol in the saliva. Cortisol diffuses passively, and its concentration is not affected by the rate of saliva production. Changes in plasma concentrations are reflected within a few minutes in the saliva, which makes Csalmeasurement a good indicator of adrenal cortisol production. Salivary levels represent approximately $10 \%$ of plasma concentrations [5-6]. Furthermore, measurement of steroids in the saliva offers several advantages over methods measuring total serum cortisol concentrations. These include a simple and noninvasive sampling procedure performed in a stress-free setting[8]. These advantages highlight the utility of saliva in certain populations such as pediatric patients, especially children with high weight, hypertension or osteoporosis, patients with psychiatric disorders or under high stress and subjects with severe renal failure [3, 9-11]. The fact that a prominent feature of CS is disruption of the circadian rhythm makes measurement of Csal at $11 \mathrm{pm}$ more relevant[4,7]. The potential value of corticosteroid measurement in the saliva was acknowledged in 1959, but the absence of an assay with adequate sensitivity $(\mathrm{Sn})$ and specificity $(\mathrm{Sp})$ hindered the implementation of such measurement [12-13]. At present, even if there are several automated methods for measurement of Csal, technologies with a high $\mathrm{Sn}$, rapid time of response in results and high analytical accuracy are required. Of all commercially available automated methods, only one has a calibration curve that may be adjusted to the low concentration at which cortisol is found in saliva, thus ensuring cortisol quantification [6-7, 14]. The aim of this study was to evaluate the analytical performance of such method (CLIA, Unicel 600 DxI Access ${ }^{\circledR}$ Beckman Coulter, Inc., U.S.A) and to find the cutoff value for the diagnosis of CS.

\section{Materials and Methods}

\subsection{Study Population}

Csal was measured in 32 healthy subjects (23 men and 9 women) under no treatment with glucocorticosteroids or other drugs and with body mass index (BMI) restrictions in both genders. Csal was measured in parallel in 9 patients $(3$ men and 6 women) recently diagnosed with CS who had not received primary treatment for their disease yet. Prior to sample collection, participants abstained for 2 hours from brushing their teeth, smoking, eating or drinking anything but water. Subjects with cuts or abrasions in their oral cavity were excluded from this study.

\subsection{Preanalytical Variables}

All samples were collected by passive drooling at $11 \mathrm{pm}$ into sterile plastic tubes with screw caps provided by the Laboratory. Once obtained, samples were stored at $2-8^{\circ} \mathrm{C}$ until delivery and then frozen at $-20^{\circ} \mathrm{C}$. At the time of analysis, samples were thawed and centrifuged at $1500 \mathrm{~g}$ for 10 minutes at room temperature in order to obtain a mucusfree supernatant.

\subsection{Method}

Csal samples were assayed using the Unicel 600 DxI Access ${ }^{\circledR}$ automated analyzer (Beckman Coulter, Inc., U.S.A). The cortisol assay is a competitive binding immunoenzymatic assay that uses rabbit anti-cortisol antibody, cortisol-alkaline phosphatase conjugate and paramagnetic particles coated with anti-rabbit capture antibody[15]. The analyte concentrations were determined from a multi-point calibration curve, adjusting the one provided by the manufacturer of the kit for determinations in serum samples. The calibration curve was adjusted to Csal concentrations, diluting all standards 1:10 (approximate final concentrations: $5.5 \mathrm{nmol} / \mathrm{L} ; 13.8 \mathrm{nmol} / \mathrm{L}$; $27.6 \mathrm{nmol} / \mathrm{L} ; 69.0 \mathrm{nmol} / \mathrm{L} ; 165.0 \mathrm{nmol} / \mathrm{L})$. Thus, the working curve was constructed for a range from 0.0 to $165.0 \mathrm{nmol} / \mathrm{L}$. It was plotted on a logit-log scale and sample signal values were interpolated into the curve obtained to determine cortisol concentrations in $\mathrm{nmol} / \mathrm{L}$. Functional sensitivity (FS) was set at $2 \mathrm{nmol} / \mathrm{L}$, as previously estimated and published in RAEM 2005[16]. Since then, FS is regularly checked at clinically relevant intervals using different reagent lots.

\subsection{Study Design: Evaluation of Validation Parameters}

\subsubsection{Linearity and Matrix Effect}

Six 6-point calibration curves were prepared from a pool of saliva samples obtained at $11 \mathrm{pm}$ from 30 healthy subjects with cortisol values below $1 \mathrm{nmol} / \mathrm{L}$. Samples were spiked with calibrators provided by the manufacturer: Access Cortisol Calibrators (final concentrations: 0.0, 5.5, 13.8, 27.6, 69.0 and $165.5 \mathrm{nmol} / \mathrm{L}$ ). In parallel, 6 curves were prepared using the commercial diluent supplied by the manufacturer: Access Wash Buffer II. All 12 curves were processed in duplicate (CLSI-EP 6 A) [17].

\subsubsection{Limit of Blank (LoB)}

It was established from repeated measurements (60 times) of a blank sample (diluent).

\subsubsection{Limit of Quantitation}

It was assessed according to the CLSI-EP-17A2 protocol [18] by total error estimation from the preparation of calibration curves using a salivary matrix with 6 levels of cortisol concentration $(0.0 ; 5.5 ; 13.8 ; 27.6,69.0$ and 165.5 $\mathrm{nmol} / \mathrm{L})$, processed in quadruplets and using two different reagent lots (Lot 1 and Lot 2). The total acceptable error (TAE) was set at $25 \%$. 


\subsubsection{Recovery Test}

Standard cortisol solution (concentrations: 5.0, 10.0 and $40.0 \mathrm{nmol} / \mathrm{L}$ ) was added to 3 aliquots of a saliva sample with a known cortisol concentration $(2.5 \mathrm{nmol} / \mathrm{L})$. Determinations were performed in triplicate. The acceptable recovery range is $80-120 \%$.

\subsubsection{Diagnostic Performance}

Through a Receiver Operating Characteristic (ROC) curve analysis using Csal values from all 9 patients with CS and 32 healthy subjects, the Snand Sp were determined and the cutoff value was established to differentiate both populations.

\subsection{Statistical Analysis}

All data were analyzed using the Kolmogorov-Smirnov test. ROC curve analysis was performed using the MedCalc Statistical Software version 12.7.7.

\section{Results}

\subsection{Linearity and Matrix Effect}

The results obtained showed that the assay evaluated was linear between $1.8 \mathrm{nmol} / \mathrm{L}$ and $108.0 \mathrm{nmol} / \mathrm{L}\left(\mathrm{R}^{2}=0.997\right)$.

When comparing matrices, no significant differences were found between the calibration curves with diluent matrix (1.833, range: -1.961 to -1.704$)$ and salivary matrix $(-1.824$, range: -2.068 to -1.582 ). Figure 1 .

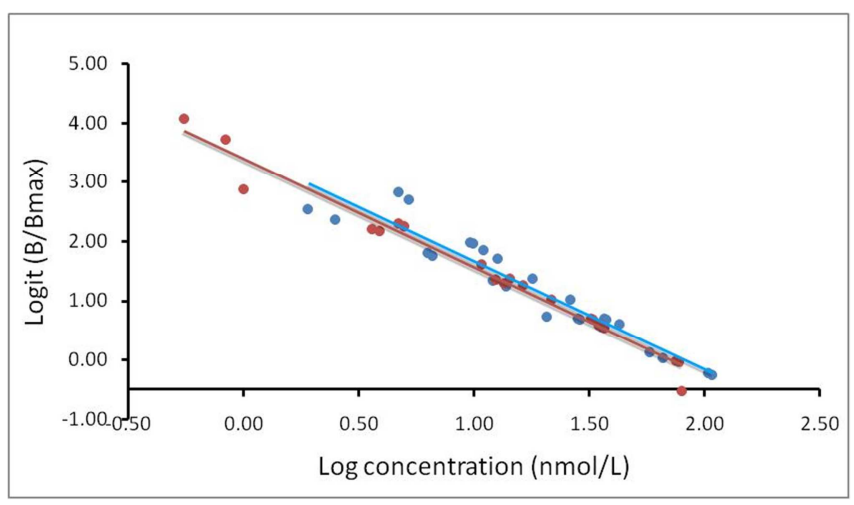

Figure 1. Comparison of calibration curves using salivary matrix (blue) and buffer (red). There is overlapping of both curves.

\subsection{Limit of Blank}

Data obtained showed nonparametric distribution. The limit of blank (LoB) obtained was $0.1 \mathrm{nmol} / \mathrm{L}$, established as the 95 th percentile from the repeated measurements series.

\subsection{Limit of Quantitation}

For each calibrator concentration, the total error was estimated with each reagent lot. The equations of the curves obtained are: Lot 1: $y=-0.078 x+25.14$ and Lot 2: $y=-$ $0.101 \mathrm{x}+25.04$. A cutoff value of $1.8 \mathrm{nmol} / \mathrm{L}$ was obtained for Lot 1 and of $0.4 \mathrm{nmol} / \mathrm{L}$ for Lot 2 . The limit of quantitation (LoQ) is established from the highest value obtained $(1.8 \mathrm{nmol} / \mathrm{L})$.

\subsection{Recovery Test}

Recovery of standards $(5 \mathrm{nmol} / \mathrm{L}, 10 \mathrm{nmol} / \mathrm{L}, 40 \mathrm{nmol} / \mathrm{L})$ in a pool of salivary samples of cortisol concentration yielded the following results: $102 \%, 107 \%$, and $115 \%$, respectively.

\subsection{Diagnostic Performance}

The median Csal level at $11 \mathrm{pm}$ of the 32 healthy subjects was $2.0 \mathrm{nmol} / \mathrm{L}$ with a range between $<2$ and $9 \mathrm{nmol} / \mathrm{L}$. All 9 patients with CS had a median cortisol value of $30.3 \mathrm{nmol} / \mathrm{L}$ and a range from 15.4 to $70.0 \mathrm{nmol} / \mathrm{L}$. The ROC curve analysis showed an area under the curve equal to 1 (p $<0.0001)$ and a cutoff value of $9.0 \mathrm{nmol} / \mathrm{L}$ with a $\mathrm{Sn}$ of $100 \%$ (95\% CI: 66.4-100.0) and a Sp of 100\% (95\% CI: 89.1 100.0). Figure 2.

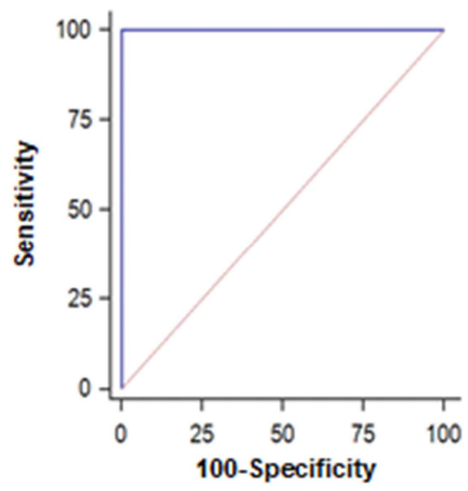

Figure 2. Receiver Operating Characteristic (ROC); curve obtained from 32 healthy subjects and 9 patients with CS. The area under the curve was 1.00 (Sn: 100\%; 95\% CI: 66.4-100.0 - Sp: 100\%; 95\% CI: 89.1-100.0).

\section{Discussion}

In recent years, measurement of steroids in saliva has demonstrated a special utility. Cortisol, as all other steroids, diffuses freely into saliva and represents the biologically active free fraction. Sample collection is simple and may be self-performed by the patient at home in a stress-free setting, in contrast to venipuncture. It has been demonstrated that factors such as smoking, eating and blood contamination due to teeth-brushing may increase Csal concentrations [19-20]. In this study, we controlled these variables by providing the subjects with instructions for proper sampling. As regards sample preservation, the high chemical stability of steroid hormones under different environmental conditions is widely recognized. No changes have been recorded in the values of samples stored at $4^{\circ} \mathrm{C}$ for up to 3 months [21].

Cortisol determination in saliva at $11 \mathrm{pm}$ is recommended by the Endocrine Society consensus guidelines for the diagnosis of CS [1]. Raff et al. were the first to report their protocol, which consisted in measuring Csal by adapting a coated-tube radioimmunoassay. Their modification of certain working conditions of the assay included increasing the sample volume to be used and the incubation time, and adjusting the kit calibrators by diluting them 1:10 in distilled water to achieve a linear curve in the expected Csal concentration [22]. In recent years, automated measurement systems have allowed 
processing a large number of samples, obtaining rapid and accurate results, with good analytical performance.

The automated chemiluminescent immunoassay (CLIA), Siemens Immulite 2000 analyzer has been recently validated for the measurement of salivary cortisol by Repetto et al. [8]. In addition, the electrochemiluminescent immunoassay (ECLIA) Cobas 6000-Cobas-e601 has been validated by the manufacturer for cortisol determination in saliva. Our laboratory has used the Unicel 600 DXI Beckman Coulter system for salivary cortisol measurement for over a decade. At first, the system was evaluated and correlated on the basis of determinations by an adapted radioimmunoassay (RIA), with very good results [23]. In this study, our aim was to evaluate the performance of the Unicel 600 DXI Beckman Coulter system following the recommendations provided by the guidelines [17-18] and retrospectively reviewing our own experience. It is worth mentioning that an advantage of this automated analyzer over the others is the availability of 6 standards and their dilutions to one-tenth for calibration of the assay, covering the whole working range(from 0.0 to $165.0 \mathrm{nmol} / \mathrm{L}$ ). This enhances its performance as compared to other platforms. The results obtained demonstrated the excellent performance of the Unicel 600 DXI Beckman Coulter automated chemoluminescent method for the measurement of salivary cortisol. Other authors have demonstrated a good correlation between serum and Csaldeterminations by the same methodology [24].

The experiment designed clearly shows an absence of matrix effect in saliva samples. It is worth noting the low FS of the assay, $2 \mathrm{nmol} / \mathrm{L}$, which we have been reporting since 2005[16]. As regards the establishment of cutoff values, many studies have reported different cutoff values depending on the method used and on the characteristics of the subjects evaluated. The first report was published by Raff et al., who suggested a value $\geq 3.6 \mathrm{nmol} / \mathrm{L}$ (1998; RIA). Findling and Raff later proposed levels above $8.6 \mathrm{nmol} / \mathrm{L}$ (2006; ELISA) and $7 \mathrm{nmol} / \mathrm{L}$ (2003; enzyme-linked immunosorbent assay) as highly likely to be diagnostic of CS [25-26]. Using an automated chemoluminescent method (2015; Cobas e411Roche), Garrido et al.., from Chile, suggested a cutoff value of $8.55 \mathrm{nmol} / \mathrm{L}$. Our group at the School of Pharmacy and Biochemistry of the University of Buenos Aires reported a cutoff value of $5.7 \mathrm{nmol} / \mathrm{L}$ for the automated chemoluminescent immunoassay (CLIA Siemens Immulite 2000 analyzer, showing a Sn of $90.5 \%$ and a Sp of $94 \%$ [8].

Different cutoff values have been proposed in the literature for nocturnal Csal determination. In this study, we evaluated the ability of cortisol determination at $11 \mathrm{pm}$ to differentiate between healthy subjects and those with CS. A cutoff value of $9 \mathrm{nmol} / \mathrm{L}$ was obtained with a $\mathrm{Sn}$ of $100 \%$ and a $\mathrm{Sp}$ of $100 \%$ analyzed by ROC curves, with none of the healthy subjects showing results above the reference range obtained, and none of the patients with CS showing values below the proposed cutoff value. A modification of the calibration curve provided by the manufacturer has made it possible to obtain a high-sensitivity analytical performance in values close to the cutoff point, which allowed a perfect differentiation between the subjects of both populations. The strength of this study lies in the fact that there are no similar studies in the literature for this platform.

\section{Conclusion}

We conclude that the method used offers an excellent analytical performance. Measurement of Csal at $11 \mathrm{pm}$ by the Access Beckman Coulter automated chemoluminescent method is a valuable, quick and simple biochemical tool both for screening suspected populations for CS and for the diagnosis and follow-up of this condition.

\section{Ethical Responsibilities}

Protection of human subjects and animals: the authors declare that no experiments were performed on humans or animals for this investigation.

Confidentiality of data: the authors declare that they have followed the protocols of their work center on the publication of patient data.

Right to privacy and informed consent: the authors have obtained the informed consent of the patients mentioned in the article.

\section{References}

[1] Lynnette K. Nieman, Beverly M. K. Biller, James W. Findling, John Newell-Price, Martin O. Savage, Paul M. Stewart, Victor M. Montori; The Diagnosis of Cushing's Syndrome: An Endocrine Society Clinical Practice Guideline, The Journal of Clinical Endocrinology \& Metabolism. 2008; 93 (5): 15261540 .

[2] Yaneva, M; Mosnier-Pudar, H; Dugué, M; Grabar, S; Fulla, I; Bertanga, X. Midnigthsalivary cortisol for the initial diagnosis of Cushing's Syndrome of various causes. J ClinEndocrinolMetabol.2004; 89 (7): (3346-3351).

[3] Lopez Mondéjar, P;Fuentes, M; Mauri, M; Mora, A; Pérez Soto, M; Vargas, F; Hidalgo, A.Determinación de cortisol salival en el diagnóstico de Cushing pediátrico. An. Pediatr. Barcelona. 2006; 64 (3): (270-272).

[4] Kaufman E; Lamster B. The diagnostic applications of saliva. Crit. Rev. Oral Biol. Med.; 2002; 13 (2): 197-212.

[5] Gröschl, M. Current status of salivary hormone analysis. Clinical Chemistry. 2008; 54:11 (1759-1769).

[6] Inder W; Dimeski G; Russell A. Measurement of salivary cortisol in 2012. Laboratorytechniques and clinicalindications. Clin. Endocrinol. (oxf); 2012; 77: (645-651).

[7] Vining, R.; Mcginley, R.; Maksvytis, J.; Ho, K. Salivary cortisol: a better measure of adrenal cortical function than serum cortisol. Ann Clin Biochem 1983; 20 (329-335).

[8] Repetto E; González D; Jacobsen D; Smithuis F; Jamardo J; Cano M; Aranda C; Oneto A; Berg G; Fabre B. Evaluation of anautomated chemiluminescent immunoassay forsalivary cortisol measurement. Utility in the diagnosis of Cushing's syndrome. Clin. Chem. Lab. Med.; 2016, aop. 
[9] Garde, A.; Hansen, A. Long-termstability of salivary cortisol. Scandinavian Journal of Clinical and Laboratory Investigation. 2005; 65:5: (433-436).

[10] Fabre B.; MeschV; Oneto A; Macalini G; Grosman H; Aranda C; BergG.La saliva y su utilidad en la evaluación de la función endocrinológica. Revista SAEGRE,2009;vol XVI; no3: (26-43).

[11] Lepez M; Caamaño E; Romero C; Fiedler Y; Araya V. Determinación de los niveles de cortisol salival en una muestra de sujetos de Santiago de Chile. Rev. Med. Chile; 2010; vol 138; $\mathrm{n}^{\circ} 2:(168-174)$.

[12] Raff, H. Utility of salivary cortisol measurements in Cushing's Syndrome and adrenal insufficiency. Clin Endocrinol Metab. 2009; 94(10): (3647-3655).

[13] Katz, F.; Shannon, I. Adrenal corticosteroids in submaxillaryfluids. J Dental Research. 1969; 48(448-451).

[14] Walker, R.; Riad-Fahmy, D.; Read, G. Adrenal status assessed by directradioimmunoassay of cortisol in whole saliva orparotid saliva. Clin Chem. 1978; 24: (1460-1463).

[15] Beckman Coulter. Acess Immunoassay Systems. Access Cortisol. 2015.

[16] Fabre, B; Oneto, A; Schonfeld, C; Belli, S; Aranda, C. Evaluación del comportamiento de tres inmunoensayos (IE) en la medición de cortisol en saliva en una población normal. Revista Argentina de Endocrinología RAEM 2005; vol 42, n suplementario, (p. 133).

[17] CLSI. Evaluation of the Linearity of Quantitative Measurement Procedures: A Statistical Approach, 1st Edition. CLSI document EP06-A. Wayne, PA: Clinical and Laboratory Standards Institute; 2003.

[18] CLSI. Evaluation of Detection Capability for Clinical
Laboratory Measurement Procedures; Approved GuidelineSecond Edition. CLSI document EP17- A2. Wayne, PA: Clinical and Laboratory Standards Institute; 2012.

[19] Papanicolaou, D; Mullen, N; Kyrou, I; Nieman, L. Nightimesalivary cortisol: a useful test for the diagnosis of Cushing's Syndrome. J Clin Endocrinol Metabol. 2002; 87 (10); (4515-4521).

[20] Badrick E, Kirschbaum C, Kumasi M. The Relationship between Smoking Status and Cortisol Secretion. J Clin Endocrinol Metab. 2007; 92: (819-824).

[21] Kivlighan KT, Granger DA, Schwartz EB, Nelson V, Curran M, Shirtcliff EA. Quantifying blood leakage into the oral mucosa and its effects on the measurement of cortisol, dehydroepiandrosterone, and testosterone in saliva. Horm Behav 2004; 46: (39-44).

[22] Raff, H; Raff, J; Findling, JW. Late-Night Salivary Cortisol as a Screening Test for Cushing's syndrome. Journal of Clinical Endocrinology and Metabolism. 1998; 83(8); (2681-2686).

[23] Garde AH, Hansen AM. Long-termstability of salivary cortisol. Scand J ClinLab Invest. 2005; 65: (433-436).

[24] Forclaz, MV; Morato, E; Pennisi, A; Falco, S; Olsen, G; Rodríguez, P; Papazian, R; Bergadá, I. Niveles de cortisol en la saliva y séricos en recién nacidos. Arch Argent Pediatr 2017; 115 (3): 262-266.

[25] Raff, H; Findling, JW. A physiologicalapproach to diagnosis of Cushing'ssyndrome. Ann InternMed. 2003; 138: (980-991).

[26] Garrido, MP; Romero, CO; Cid, MPR; Araya, VQ. Detección de cortisol salival nocturno en una muestra de sujetos de Santiago de Chile, mediante la técnica de electroquimioluminiscencia. Rev. Chil. Endocrinol. Diabetes; 2015; 8 (3): (102-107). 